SUPPORTING INFORMATION

\title{
Novel Synthesis of bicycles with fused pyrrole, indole, oxazole and imidazole rings.
}

Alan R. Katritzky, * Sanjay K. Singh and Sergey Bobrov

Center for Heterocyclic Compounds, University of Florida, Department of Chemistry,

Gainesville, Florida 32611-7200, USA

e-mail: Katritzky@chem.ufl.edu

$\underline{\text { Table of Contents }}$

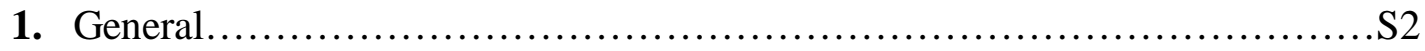

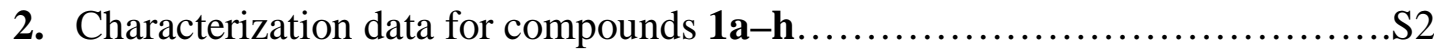

3. Characterization data for compounds $\mathbf{2} \mathbf{a}-\mathbf{h}$ and $\mathbf{1 2} \ldots \ldots \ldots \ldots \ldots \ldots \ldots \ldots \ldots \ldots$

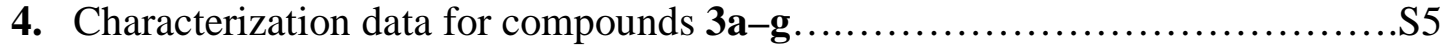

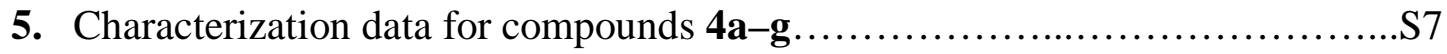




\section{General.}

Melting points were determined on a hot-stage apparatus and are uncorrected. All NMR spectra were recorded in $\mathrm{CDCl}_{3}$, with TMS as the internal standard for ${ }^{1} \mathrm{H}(300$ $\mathrm{MHz})$ or the solvent as the internal standard for ${ }^{13} \mathrm{C}(75 \mathrm{MHz})$. THF was dried over sodium/benzophenone and used freshly distilled. Column chromatography was conducted on silica gel 200-425 meshes.

\section{Characterization data for compounds 1a-h.}

3,3-Dimethyl-1H-pyrrolo[1,2-c]oxazol-1-one (1a): white needles from methanol (80\%), mp 52-53 ${ }^{\circ} \mathrm{C} ;{ }^{1} \mathrm{H}$ NMR: $\delta 6.95$ (br s, $\left.1 \mathrm{H}\right), 6.73(\mathrm{~d}, J=3.6 \mathrm{~Hz}, 1 \mathrm{H}), 6.51(\mathrm{dd}, J=$ 3.6, $2.1 \mathrm{~Hz}, 1 \mathrm{H}), 1.80(\mathrm{~s}, 6 \mathrm{H}) ;{ }^{13} \mathrm{C} \mathrm{NMR}: \delta 159.1,120.3,117.0,116.9,108.6,93.7,28.2$. Anal. Calcd for $\mathrm{C}_{8} \mathrm{H}_{9} \mathrm{NO}_{2}$ : C, 63.56; H, 6.00; N, 9.27. Found: C, 63.50; H, 6.17; N, 9.14. (士)-3-Isopropylpyrrolo[1,2-c $]$ oxazol-1-one (1b): colorless oil $(98 \%) ;{ }^{1} \mathrm{H}$ NMR: $\delta 6.99$ $(\mathrm{d}, J=1.7 \mathrm{~Hz}, 1 \mathrm{H}), 6.75(\mathrm{~d}, J=3.7 \mathrm{~Hz}, 1 \mathrm{H}), 6.53-6.51(\mathrm{~m}, 1 \mathrm{H}), 5.91(\mathrm{~d}, J=3.2 \mathrm{~Hz}, 1 \mathrm{H})$, 2.28 (septet of a doublet, $J=6.9,3.2 \mathrm{~Hz}, 1 \mathrm{H}), 1.08(\mathrm{~d}, J=6.9 \mathrm{~Hz}, 3 \mathrm{H}), 0.83(\mathrm{~d}, J=7.0$ $\mathrm{Hz}, 3 \mathrm{H}) ;{ }^{13} \mathrm{C}$ NMR: $\delta 159.8,120.8,118.5,116.9,108.2,91.8,33.5,16.1,13.9$. Anal. Calcd for $\mathrm{C}_{9} \mathrm{H}_{11} \mathrm{NO}_{2}$ : C, 65.44; H, 6.71; N, 8.48. Found: C, 65.47; H, 7.11; N, 8.37.

Spiro[cyclohexane-1,3'-[1H,3H]pyrrolo[1,2-c]oxazol-1-one] (1c): white needles (80\%), mp 99-100 ${ }^{\circ} \mathrm{C} ;{ }^{1} \mathrm{H}$ NMR: $\delta 6.94(\mathrm{~d}, J=2.3 \mathrm{~Hz}, 1 \mathrm{H}), 6.73(\mathrm{~d}, J=4.3 \mathrm{~Hz}, 1 \mathrm{H})$, $4.49(\mathrm{dd}, J=4.3,2.3 \mathrm{~Hz}, 1 \mathrm{H}), 1.99-1.78(\mathrm{~m}, 9 \mathrm{H}), 1.44-1.37(\mathrm{~m}, 1 \mathrm{H}) ;{ }^{13} \mathrm{C} \mathrm{NMR}: \delta 159.4$ 120.2, 117.3, 116.4, 108.2, 94.9, 37.9, 24.0, 22.4. Anal. Calcd for $\mathrm{C}_{11} \mathrm{H}_{13} \mathrm{NO}_{2}$ : C, 69.09; H, 6.85; N, 7.32. Found: C, 69.29; H, 6.95; N, 7.26. 
( \pm )-3-p-Tolylpyrrolo[1,2-c]oxazol-1-one (1d): white needles from methanol (76\%), mp $143-144{ }^{\circ} \mathrm{C} ;{ }^{1} \mathrm{H}$ NMR: $\delta 7.24(\mathrm{~d}, J=8.0 \mathrm{~Hz}, 2 \mathrm{H}), 7.16(\mathrm{~d}, J=8.0 \mathrm{~Hz}, 2 \mathrm{H}), 6.84-6.81$ (m, 3H), 6.54-6.52 (m, 1H), 2.39 (s, 3H); ${ }^{13} \mathrm{C}$ NMR: $\delta$ 159.3, 141.2, 131.7, 129.9, 126.7, 120.7, 118.9, 117.4, 108.7, 88.6, 21.3. Anal. Calcd for $\mathrm{C}_{13} \mathrm{H}_{11} \mathrm{NO}_{2}$ : C, 73.22; $\mathrm{H}, 5.20 ; \mathrm{N}$, 6.57. Found: C, 73.04; H, 5.09; N, 6.60.

Spiro[cyclopentane-1,3'-[1H,3H]pyrrolo[1,2-c] $]$ oxazol-1-one] (1e): colorless oil (81\%); ${ }^{1} \mathrm{H}$ NMR: $\delta 6.96(\mathrm{~d}, J=2.1 \mathrm{~Hz}, 1 \mathrm{H}), 6.72(\mathrm{~d}, J=3.7 \mathrm{~Hz}, 1 \mathrm{H}), 6.52(\mathrm{dd}, J=3.7$, $2.5 \mathrm{~Hz}, 1 \mathrm{H}), 2.33-2.24(\mathrm{~m}, 2 \mathrm{H}), 2.18-1.94(\mathrm{~m}, 6 \mathrm{H}) ;{ }^{13} \mathrm{C} \mathrm{NMR}: \delta 159.1,120.9,117.1$, 116.7, 108.2, 102.9, 39.6, 23.5. Anal. Calcd for $\mathrm{C}_{10} \mathrm{H}_{11} \mathrm{NO}_{2}$ : C, 67.78; $\mathrm{H}, 6.26 ; \mathrm{N}, 7.90$. Found: C, 67.50; H, 6.28; N, 8.34.

3,3-Diethylpyrrolo[1,2-c]oxazol-1-one (1f): white microcrystals (80\%), mp 39-41 ${ }^{\circ} \mathrm{C}$; ${ }^{1} \mathrm{H}$ NMR: $\delta 6.85(\mathrm{~d}, J=1.8 \mathrm{~Hz}, 1 \mathrm{H}), 6.72(\mathrm{~d}, J=3.6 \mathrm{~Hz}, 1 \mathrm{H}), 6.57(\mathrm{dd}, J=3.6,2.4 \mathrm{~Hz}$, 1H), 2.22-2.10 (m, 2H), 2.04-1.91 (m, 2H), $0.73(\mathrm{t}, J=7.3 \mathrm{~Hz}, 6 \mathrm{H}) ;{ }^{13} \mathrm{C}$ NMR: $\delta$ 160.0, 122.2, 117.1, 116.9, 108.0, 98.1, 32.3, 6.2. Anal. Calcd for $\mathrm{C}_{10} \mathrm{H}_{13} \mathrm{NO}_{2}$ : C, 67.02; H, 7.31; N, 7.82. Found: C, 66.84; H, 7.03; N, 7.68.

( \pm )-3-Furan-2-yl-pyrrolo[1,2-c]oxazol-1-one (1g): white needles from methanol (64\%), mp $76-77{ }^{\circ} \mathrm{C} ;{ }^{1} \mathrm{H}$ NMR: $\delta 7.50(\mathrm{dd}, J=1.8,0.8 \mathrm{~Hz}, 1 \mathrm{H}), 6.94(\mathrm{ddd}, J=2.5,0.9$, $0.7 \mathrm{~Hz}, 1 \mathrm{H}), 6.91(\mathrm{~s}, 1 \mathrm{H}), 6.85(\mathrm{dd}, J=3.8,1.0 \mathrm{~Hz}, 1 \mathrm{H}), 6.57(\mathrm{ddd}, J=3.4,0.8,0.4 \mathrm{~Hz}$, $1 \mathrm{H}), 6.56(\mathrm{dd}, J=3.8,2.5 \mathrm{~Hz}, 1 \mathrm{H}), 6.44(\mathrm{dd}, J=1.8 \mathrm{~Hz}, 1 \mathrm{H}) ;{ }^{13} \mathrm{C} \mathrm{NMR}: \delta 158.7,146.6$, 145.0, 120.2, 119.2, 117.5, 111.6, 110.8, 109.1, 81.5. Anal. Calcd for $\mathrm{C}_{10} \mathrm{H}_{7} \mathrm{NO}_{3}$ : C, 63.49; H, 3.73; N, 7.40. Found: C, 63.23; H, 3.68; N, 7.23.

( \pm )-3-Methyl-3-phenylpyrrolo[1,2-c $]$ oxazol-1-one (1h): colorless oil $(47 \%) ;{ }^{1} \mathrm{H}$ NMR: $\delta 7.46-7.33(\mathrm{~m}, 5 \mathrm{H}), 6.98(\mathrm{dd}, J=2.3,1.0 \mathrm{~Hz}, 1 \mathrm{H}), 6.80(\mathrm{~d}, J=3.8 \mathrm{~Hz}, 1 \mathrm{H}), 6.54$ 
(dd, $J=3.7,2.6 \mathrm{~Hz}, 1 \mathrm{H}), 2.17$ (s, 3H); ${ }^{13} \mathrm{C}$ NMR: $\delta$ 158.9, 138.5, 129.7, 128.9, 124.8, 120.7, 118.3, 117.3, 108.8, 94.7, 27.6. Anal. Calcd for $\mathrm{C}_{13} \mathrm{H}_{11} \mathrm{NO}_{2}$ : C, 73.22; H, 5.20; $\mathrm{N}$, 6.57. Found: C, 73.11; H, 5.34; N, 6.73.

\section{Characterization data for compounds $2 \mathrm{a}-\mathrm{d}$ and 12.}

3,3-Dimethyloxazolo[3,4-a]indol-1-one (2a): white needles from methanol (50\%), mp 81-82 ${ }^{\circ} \mathrm{C} ;{ }^{1} \mathrm{H}$ NMR: $\delta 7.79(\mathrm{~d}, J=8.1 \mathrm{~Hz}, 1 \mathrm{H}), 7.41-7.39(\mathrm{~m}, 2 \mathrm{H}), 7.26-7.21(\mathrm{~m}, 1 \mathrm{H})$, 7.07 (s, 1H), 1.93 (s, 6H); ${ }^{13} \mathrm{C}$ NMR: $\delta 160.0,132.6,131.6,125.3,124.8,124.1,121.5$ 110.3, 100.9, 94.7, 27.0. Anal. Calcd for $\mathrm{C}_{12} \mathrm{H}_{11} \mathrm{NO}_{2}: \mathrm{C}, 71.63 ; \mathrm{H}, 5.51 ; \mathrm{N}, 6.96$. Found: C, 71.50; H, 5.37; N, 6.94.

( \pm )-3-Isopropyloxazolo[3,4-a]indol-1-one (2b): white plates from methanol (83\%), mp $118-119^{\circ} \mathrm{C} ;{ }^{1} \mathrm{H}$ NMR: $\delta 7.80(\mathrm{~d}, J=8.2 \mathrm{~Hz}, 1 \mathrm{H}), 7.40-7.37(\mathrm{~m}, 2 \mathrm{H}), 7.28-7.22(\mathrm{~m}$, 1H), $7.09(\mathrm{~s}, 1 \mathrm{H}), 6.22(\mathrm{~d}, J=2.3 \mathrm{~Hz}, 1 \mathrm{H}), 2.63($ septet of a doublet, $J=7.0,2.3 \mathrm{~Hz}, 1 \mathrm{H}$ ), $1.27(\mathrm{~d}, J=7.0 \mathrm{~Hz}, 3 \mathrm{H}), 0.63(\mathrm{~d}, J=7.0 \mathrm{~Hz}, 3 \mathrm{H}) ;{ }^{13} \mathrm{C}$ NMR: $\delta 160.9,132.6,132.5$, 125.3, 125.1, 124.2, 121.8, 110.7, 101.1, 92.2, 32.8, 17.5, 12.7. Anal. Calcd for $\mathrm{C}_{13} \mathrm{H}_{13} \mathrm{NO}_{2}: \mathrm{C}, 72.54 ; \mathrm{H}, 6.09 ; \mathrm{N}, 6.51$. Found: C, 72.50; H, 6.22; N, 6.38.

Spiro[cyclohexane-1,3'-[1H,3H]oxazolo[3,4-a]indol-1-one] (2c): colorless oil (16\%);

${ }^{1} \mathrm{H}$ NMR: $\delta 7.80(\mathrm{~d}, J=8.1 \mathrm{~Hz}, 1 \mathrm{H}), 7.45-7.36(\mathrm{~m}, 2 \mathrm{H}), 7.26-7.21(\mathrm{~m}, 1 \mathrm{H}), 7.07$ (s, 1H), $2.37-2.26(\mathrm{~m}, 2 \mathrm{H}), 1.97-1.87(\mathrm{~m}, 6 \mathrm{H}), 1.58(\mathrm{~s}, 1 \mathrm{H}), 1.52-1.34(\mathrm{~m}, 1 \mathrm{H}) ;{ }^{13} \mathrm{C}$ NMR: $\delta$ 161.3, 132.7, 132.6, 125.3, 125.1, 124.3, 121.5, 110.7, 100.9, 96.1, 36.8, 24.4, 22.6. Anal. Calcd for $\mathrm{C}_{15} \mathrm{H}_{15} \mathrm{NO}_{2}:$ C, 74.67; H, 6.27; N, 5.80. Found: C, 74.50; H, 6.17; N, 6.10.

(土)-3-p-Tolyloxazolo[3,4-a $]$ indol-1-one (2d): white needles from methanol (30\%), mp 143-144 ${ }^{\circ} \mathrm{C} ;{ }^{1} \mathrm{H}$ NMR: $\delta 7.80-7.76(\mathrm{~m}, 1 \mathrm{H}), 7.23-7.16(\mathrm{~m}, 7 \mathrm{H}), 6.96(\mathrm{~s}, 1 \mathrm{H}), 6.95-6.92$ 
(m, 1H), 2.36 (s, 3H); ${ }^{13} \mathrm{C}$ NMR: $\delta 160.3,141.4,132.7,132.6,130.6,129.9,127.0,125.4$, 125.0, 124.0, 121.9, 110.9, 101.5, 89.0, 21.3. Anal. Calcd for $\mathrm{C}_{17} \mathrm{H}_{13} \mathrm{NO}_{2}$ : C, 77.55; $\mathrm{H}$, 4.98; N, 5.32. Found: C, 77.19; H, 4.99; N, 5.30.

$1 H$-Indole-2-carboxylic acid benzotriazol-1-yl-p-tolylmethyl ester (12): white needles from methanol (23\%), mp $195-196{ }^{\circ} \mathrm{C} ;{ }^{1} \mathrm{H}$ NMR: $\delta 9.30$ (s, 1H), $8.73(\mathrm{~s}, 1 \mathrm{H})$, $8.07(\mathrm{~d}, J=8.1 \mathrm{~Hz}, 1 \mathrm{H}), 7.67(\mathrm{~d}, J=8.1 \mathrm{~Hz}, 1 \mathrm{H}), 7.50(\mathrm{~d}, J=8.0 \mathrm{~Hz}, 1 \mathrm{H}), 7.44-7.29(\mathrm{~m}$, 7H), 7.25-7.20 (m, 2H), 7.17-7.12 (m, 1H), $2.36(\mathrm{~s}, 3 \mathrm{H}) ;{ }^{13} \mathrm{C}$ NMR: $\delta$ 159.9, 139.8, 137.5, 131.9, 131.0, 129.6, 128.1, 127.2, 126.2, 126.1, 125.3, 124.4, 122.7, 121.2, 120.2, 112.1, 110.7, 110.6, 80.9, 21.2. Anal. Calcd for $\mathrm{C}_{23} \mathrm{H}_{18} \mathrm{~N}_{4} \mathrm{O}_{2}: \mathrm{C}, 72.24 ; \mathrm{H}, 4.74 ; \mathrm{N}, 14.65$. Found: C, 72.11; H, 4.75; N, 14.49 .

\section{Characterization data for compounds 3a-g.}

2-Benzyl-3-thioxo-2,3-dihydropyrrolo[1,2-c]imidazol-1-one (3a): yellow needles from methanol (41\%), mp $75-76{ }^{\circ} \mathrm{C} ;{ }^{1} \mathrm{H}$ NMR: $\delta 7.46-7.43(\mathrm{~m}, 2 \mathrm{H}), 7.38(\mathrm{dd}, J=3.0$, $0.9 \mathrm{~Hz}, 1 \mathrm{H}), 7.35-7.27(\mathrm{~m}, 3 \mathrm{H}), 6.77(\mathrm{dd}, J=3.4,0.8 \mathrm{~Hz}, 1 \mathrm{H}), 6.38(\mathrm{t}, J=3.2 \mathrm{~Hz}, 1 \mathrm{H})$, $5.02(\mathrm{~s}, 2 \mathrm{H}) ;{ }^{13} \mathrm{C}$ NMR: $\delta 174.2,158.5,135.4,128.7,128.6,128.0,123.1,119.8,117.3$, 114.2, 44.5. Anal. Calcd for C13H10N2OS: C, 64.44; H, 4.16; N, 11.56. Found: C, 64.63; H, 4.12; N, 11.45 .

2-Phenyl-3-thioxo-2,3-dihydropyrrolo[1,2-c $]$ imidazol-1-one ${ }^{\text {i) }}$ (3b): yellow needles from methanol (26\%), mp $138-139{ }^{\circ} \mathrm{C} ;{ }^{1} \mathrm{H}$ NMR: $\delta 7.58-7.43(\mathrm{~m}, 4 \mathrm{H}), 7.39-7.36(\mathrm{~m}$, 2H), $6.87(\mathrm{dd}, J=3.6,0.9 \mathrm{~Hz}, 1 \mathrm{H}), 6.47(\mathrm{t}, J=3.2 \mathrm{~Hz}, 1 \mathrm{H}) ;{ }^{13} \mathrm{C} \mathrm{NMR}: \delta 174.2,158.0$,

\footnotetext{
i) Papadopoulos, E. P. J. Org. Chem. 1966, 31, 3060. Budhram, R. S.; Uff, B. C.; Jones, R. A.; Jones, R. O. Org. Magn. Reson. 1980, 13(2), 89.
} 
132.3, 129.3, 129.2, 128.3, 122.8, 120.0, 117.7, 114.4. Anal. Calcd for $\mathrm{C}_{12} \mathrm{H}_{8} \mathrm{~N}_{2} \mathrm{OS}$ : C, 63.14; H, 3.53; N, 12.27. Found: C, 63.27; H, 3.40; N, 12.07 .

2-Ethyl-3-thioxo-2,3-dihydropyrrolo[1,2-c]imidazol-1-one (3c): yellow needles from methanol (77\%), mp 60-61 ${ }^{\circ} \mathrm{C} ;{ }^{1} \mathrm{H}$ NMR: $\delta 7.39(\mathrm{~d}, J=2.9 \mathrm{~Hz}, 1 \mathrm{H}), 6.76(\mathrm{~d}, J=$ $3.4 \mathrm{~Hz}, 1 \mathrm{H}), 6.38(\mathrm{t}, J=3.2 \mathrm{~Hz}, 1 \mathrm{H}), 3.91(\mathrm{q}, J=7.1 \mathrm{~Hz}, 2 \mathrm{H}), 1.28(\mathrm{t}, J=7.1 \mathrm{~Hz}, 3 \mathrm{H})$;

${ }^{13}$ C NMR: $\delta 174.3,158.4,123.3,119.6,117.1,113.8,36.3,13.1$. Anal. Calcd for $\mathrm{C}_{8} \mathrm{H}_{8} \mathrm{~N}_{2} \mathrm{OS}: \mathrm{C}, 53.31 ; \mathrm{H}, 4.47 ; \mathrm{N}, 15.54$. Found: C, 53.33; H, 4.37; N, 15.27.

2-Isopropyl-3-thioxo-2,3-dihydropyrrolo[1,2-c]imidazol-1-one (3d): yellow needles from methanol (30\%), mp $70-71{ }^{\circ} \mathrm{C} ;{ }^{1} \mathrm{H}$ NMR: $\delta 7.36(\mathrm{~d}, J=2.8 \mathrm{~Hz}, 1 \mathrm{H}), 7.26(\mathrm{~s}, 1 \mathrm{H})$, $6.72(\mathrm{~d}, J=2.8 \mathrm{~Hz}, 1 \mathrm{H}), 6.37(\mathrm{t}, J=3.1 \mathrm{~Hz}, 1 \mathrm{H}), 4.83($ septet, $J=7.0 \mathrm{~Hz}, 1 \mathrm{H}), 1.52(\mathrm{~d}, J$ $=7.0 \mathrm{~Hz}, 6 \mathrm{H}) ;{ }^{13} \mathrm{C}$ NMR: $\delta 174.5,158.8,123.2,119.6,117.0,113.4,47.5,19.5$. Anal. Calcd for $\mathrm{C}_{9} \mathrm{H}_{10} \mathrm{~N}_{2} \mathrm{OS}$ : C, 55.65; H, 5.19; N, 14.42. Found: C, 55.88; H, 4.98; N, 14.07.

2-p-Tolylpyrrolo[1,2-c]imidazole-1,3-dione (3e): white needles from methanol (71\%), mp 194-195 ${ }^{\circ} \mathrm{C} ;{ }^{1} \mathrm{H}$ NMR: $\delta 7.34(\mathrm{~d}, J=2.9 \mathrm{~Hz}, 1 \mathrm{H}), 7.30(\mathrm{~s}, 4 \mathrm{H}), 6.88(\mathrm{~d}, J=$ $3.3 \mathrm{~Hz}, 1 \mathrm{H}), 6.51(\mathrm{t}, J=3.2 \mathrm{~Hz}, 1 \mathrm{H}), 2.40(\mathrm{~s}, 3 \mathrm{H}) ;{ }^{13} \mathrm{C} \mathrm{NMR}: \delta 157.5,138.6,129.9$, 128.3, 126.3, 124.9, 119.3, 117.8, 114.1, 21.1. Anal. Calcd for $\mathrm{C}_{13} \mathrm{H}_{10} \mathrm{~N}_{2} \mathrm{O}_{2}: \mathrm{C}, 69.02 ; \mathrm{H}$, 4.46; N, 12.38. Found: C, 69.07; H, 4.49; N, 12.33 .

2-Benzylpyrrolo[1,2-c imidazole-1,3-dione $^{\text {ii) }}$ (3f): white needles from methanol (75\%), mp 75-76 ${ }^{\circ} \mathrm{C} ;{ }^{1} \mathrm{H}$ NMR: $\delta$ 7.44-7.39 (m, 2H), 7.36-7.28 (m, 3H), 7.22 (dd, $J=$ 2.9, $0.8 \mathrm{~Hz}, 1 \mathrm{H}), 6.76(\mathrm{dd}, J=3.4,0.8 \mathrm{~Hz}, 1 \mathrm{H}), 6.41(\mathrm{t}, J=3.3 \mathrm{~Hz}, 1 \mathrm{H}), 4.74(\mathrm{~s}, 2 \mathrm{H}) ;{ }^{13} \mathrm{C}$

\footnotetext{
ii) Noguchi, S; Obayashi, M.; Imanishi, M; Kawai, K. Jpn. Kokai. Patent JP 47042790; Chem. Abstr. 1972, 78, 84249.
} 
NMR: $\delta 158.2,148.5,135.7,128.7,128.6,128.0,125.3,119.0,117.3,113.6,42.4$. Anal.

Calcd for $\mathrm{C}_{13} \mathrm{H}_{10} \mathrm{~N}_{2} \mathrm{O}_{2}$ : C, 69.02; H, 4.46; N, 12.38. Found: C, 69.10; H, 4.38; N, 12.25.

2-(1-Phenylethyl)pyrrolo[1,2-c]imidazole-1,3-dione $\quad(3 \mathrm{~g})$ : white needles from methanol (95\%), mp 63-64 ${ }^{\circ} \mathrm{C} ;{ }^{1} \mathrm{H}$ NMR: $\delta 7.52-7.48(\mathrm{~m}, 2 \mathrm{H}), 7.37-7.28(\mathrm{~m}, 3 \mathrm{H}), 7.19$ (dd, $J=2.9,0.7 \mathrm{~Hz}, 1 \mathrm{H}), 6.73(\mathrm{dd}, J=3.4,0.6 \mathrm{~Hz}, 1 \mathrm{H}), 6.40(\mathrm{t}, J=3.3 \mathrm{~Hz}, 1 \mathrm{H}), 5.42(\mathrm{q}$, $J=7.3 \mathrm{~Hz}, 1 \mathrm{H}), 1.91(\mathrm{~d}, J=7.4 \mathrm{~Hz}, 3 \mathrm{H}) ;{ }^{13} \mathrm{C}$ NMR: $\delta 158.2,148.2,139.6,128.5,127.8$, 127.3, 125.0, 118.7, 117.1, 113.1, 50.3, 17.3. Anal. Calcd for $\mathrm{C}_{14} \mathrm{H}_{12} \mathrm{~N}_{2} \mathrm{O}_{2}$ : C, 69.99; H, 5.03; N, 11.66. Found: C, 69.74; H, 5.01; N, 11.48.

\section{Characterization data for compounds 4a-g.}

2-Benzyl-3-thioxo-2,3-dihydroimidazo[1,5-a]indol-1-one (4a): yellow needles from methanol (62\%), mp $136-137{ }^{\circ} \mathrm{C} ;{ }^{1} \mathrm{H}$ NMR: $\delta 8.35(\mathrm{~d}, J=8.4 \mathrm{~Hz}, 1 \mathrm{H}), 7.64(\mathrm{~d}, J=7.8$ $\mathrm{Hz}, 1 \mathrm{H}), 7.50-7.46$ (m, 3H), 7.35-7.24 (m, 4H), 7.06 (s, 1H), 5.09 (s, 2H); ${ }^{13} \mathrm{C}$ NMR: $\delta$ $172.9,159.0,135.5,134.1,132.4,129.6,128.8,128.6,128.1,127.9,124.8,124.4,114.2$ 109.3, 44.1. Anal. Calcd for $\mathrm{C}_{17} \mathrm{H}_{12} \mathrm{~N}_{2} \mathrm{OS}$ : C, 69.84; H, 4.14; N, 9.58. Found: C, 69.64; H, $4.11 ; \mathrm{N}, 9.41$.

2-Phenyl-3-thioxo-2,3-dihydroimidazo[1,5-a]indol-1-one (4b): yellow needles from methanol (21\%), mp 200-201 ${ }^{\circ} \mathrm{C} ;{ }^{1} \mathrm{H}$ NMR: $\delta 8.47(\mathrm{~d}, J=8.4 \mathrm{~Hz}, 1 \mathrm{H}), 7.75(\mathrm{~d}, J=8.2$ $\mathrm{Hz}, 1 \mathrm{H}), 7.6-7.5(\mathrm{~m}, 4 \mathrm{H}), 7.43-7.36(\mathrm{~m}, 3 \mathrm{H}), 7.20(\mathrm{~s}, 1 \mathrm{H}) ;{ }^{13} \mathrm{C}$ NMR: $\delta$ 173.3, 158.7, 134.2, 132.6, 132.3, 129.8, 129.4, 129.3, 128.6, 127.9, 125.1, 124.6, 114.6, 109.6. Anal. Calcd for $\mathrm{C}_{16} \mathrm{H}_{10} \mathrm{~N}_{2} \mathrm{OS}$ : C, 69.04; H, 3.62; N, 10.06. Found: C, 69.21; H, 3.59; N, 9.97. 
2-Ethyl-3-thioxo-2,3-dihydroimidazo[1,5-a $]_{\text {indol-1-one }}{ }^{\text {iii) }}$ (4c): yellow needles from methanol (34\%), mp 110-112 ${ }^{\circ} \mathrm{C} ;{ }^{1} \mathrm{H}$ NMR: $\delta 8.39(\mathrm{~d}, J=8.4 \mathrm{~Hz}, 1 \mathrm{H}), 7.68(\mathrm{~d}, J=8.0$ Hz, 1H), $7.52(\mathrm{t}, J=7.4 \mathrm{~Hz}, 1 \mathrm{H}), 7.30(\mathrm{t}, J=7.3 \mathrm{~Hz}, 1 \mathrm{H}), 7.08(\mathrm{~s}, 1 \mathrm{H}), 3.99(\mathrm{q}, J=7.1$ $\mathrm{Hz}, 2 \mathrm{H}), 1.32(\mathrm{t}, J=7.1 \mathrm{~Hz}, 3 \mathrm{H}) ;{ }^{13} \mathrm{C}$ NMR: $\delta$ 173.0, 158.9, 134.1, 132.5, 129.5, 128.4, 124.7, 124.4, 114.2, 109.0, 100.2, 36.0, 13.2. Anal. Calcd for $\mathrm{C}_{12} \mathrm{H}_{10} \mathrm{~N}_{2} \mathrm{OS}$ : C, 62.59; $\mathrm{H}$, 4.38; N, 12.16. Found: C, 62.70; H, 4.30; N, 11.91 .

2-Methyl-3-thioxo-2,3-dihydroimidazo[1,5-a indol-1-one $^{\text {iv) }} \quad$ (4d): yellow needles from methanol (27\%), mp $185-186{ }^{\circ} \mathrm{C} ;{ }^{1} \mathrm{H}$ NMR: $\delta 8.36(\mathrm{~d}, J=8.2 \mathrm{~Hz}, 1 \mathrm{H}), 7.67(\mathrm{~d}, J=$ 8.0 Hz, 1H), 7.54-7.49 (m, 1H), 7.32-7.27 (m, 1H), 7.07 (s, 1H), $3.36(\mathrm{~s}, 3 \mathrm{H}) ;{ }^{13} \mathrm{C}$ NMR: $\delta$ 173.5, 159.1, 134.0, 132.4, 129.5, 128.3, 124.7, 124.4, 114.2, 109.2, 27.2. Anal. Calcd for $\mathrm{C}_{11} \mathrm{H}_{8} \mathrm{~N}_{2} \mathrm{OS}$ : C, 61.09; H, 3.73; N, 12.95. Found: C, 61.22; H, 3.64; N, 12.54 .

2-p-Tolylimidazo[1,5-a]indole-1,3-dione (4e): white needles from methanol (79\%), mp $158-159{ }^{\circ} \mathrm{C} ;{ }^{1} \mathrm{H}$ NMR: $\delta 7.92(\mathrm{~d}, J=8.2 \mathrm{~Hz}, 1 \mathrm{H}), 7.71(\mathrm{~d}, J=8.0 \mathrm{~Hz}, 1 \mathrm{H}), 7.53-7.49$ (m, 1H), 7.37-7.29 (m, 5H), $7.19(\mathrm{~s}, 1 \mathrm{H}), 2.41(\mathrm{~s}, 3 \mathrm{H}),{ }^{13} \mathrm{C}$ NMR: $\delta$ 158.2, 147.8, 138.6, 133.0, 132.3, 129.9, 128.6, 128.4, 128.3, 126.2, 124.3, 124.1, 113.5, 109.4, 21.2. Anal. Calcd for $\mathrm{C}_{17} \mathrm{H}_{12} \mathrm{~N}_{2} \mathrm{O}_{2}:$ C, 73.90; H, 4.38; N, 10.14. Found: C, 73.60; H, 4.31; N, 10.09.

2-Benzylimidazo[1,5-a]indole-1,3-dione (4f): white needles from methanol (81\%), mp $153-154{ }^{\circ} \mathrm{C} ;{ }^{1} \mathrm{H}$ NMR: $\delta 7.86(\mathrm{dd}, J=8.2,1.0 \mathrm{~Hz}, 1 \mathrm{H}), 7.68(\mathrm{dd}, J=8.2,1.0 \mathrm{~Hz}$, 1H), 7.51-7.46 (m, 3H), 7.38-7.28 (m, 4H), $7.12(\mathrm{~s}, 1 \mathrm{H}), 4.82(\mathrm{~s}, 2 \mathrm{H}) ;{ }^{13} \mathrm{C}$ NMR: $\delta$

\footnotetext{
iii) Voss, M. E.; Carter, P. H.; Tebben, A. J.; Scherle, P. A.; Brown, G. D.; Thompson, L. A.; Xu, M.; Lo, Y. C.; Yang, G.; Liu, R-Q.; Strzemienski, P.; Everlof, J. G.; Trzaskos, J. M.; Decicco, C. P. Bioorg. Med. Chem. Lett. 2003, 13, 533.

iv) Kutschy, P.; Suchy, M.; Dzurilla, M.; Takasugi, M.; Kovacik, V. Collect. Czech. Chem. Commun. 2000, 65, 1163.
} 
$158.8,148.6,135.7,132.9,132.2,128.8$ (2C), 128.7, 128.4, 128.1, 124.1, 124.0, 113.3, 109.0, 42.5. Anal. Calcd for $\mathrm{C}_{17} \mathrm{H}_{12} \mathrm{~N}_{2} \mathrm{O}_{2}$ : C, 73.90; H, 4.38; N, 10.14. Found: C, 73.57; $\mathrm{H}, 4.33 ; \mathrm{N}, 9.69$.

2-(1-Phenylethyl)imidazo[1,5-a]indole-1,3-dione (4g): white needles from methanol (87\%), mp 105-106 ${ }^{\circ} \mathrm{C} ;{ }^{1} \mathrm{H}$ NMR: $\delta 7.78(\mathrm{~d}, J=8.2 \mathrm{~Hz}, 1 \mathrm{H}), 7.60(\mathrm{~d}, J=8.2 \mathrm{~Hz}, 1 \mathrm{H})$, $7.54(\mathrm{~d}, J=7.4 \mathrm{~Hz}, 2 \mathrm{H}), 7.41(\mathrm{t}, J=7.2 \mathrm{~Hz}, 1 \mathrm{H}), 7.37-7.18(\mathrm{~m}, 4 \mathrm{H}), 7.01(\mathrm{~s}, 1 \mathrm{H}), 5.48$ $(\mathrm{q}, J=7.4 \mathrm{~Hz}, 1 \mathrm{H}), 1.95(\mathrm{~d}, J=7.4 \mathrm{~Hz}, 3 \mathrm{H}) ;{ }^{13} \mathrm{C}$ NMR: $\delta 158.8,148.3,139.6,132.7$, 132.1, 128.5, 128.4, 128.2, 127.9, 127.4, 123.9, 123.8, 113.2, 108.4, 50.5, 17.3. Anal. Calcd for $\mathrm{C}_{18} \mathrm{H}_{14} \mathrm{~N}_{2} \mathrm{O}_{2}$ : C, 74.47; H, 4.86; N, 9.65. Found: C, 74.48; H, 4.88; N, 9.49. 